\title{
DETECTION OF GAMMA-RAY EMISSION FROM THE ETA-CARINAE REGION
}

M. Tavani ${ }^{1,2}$, S. Sabatini ${ }^{2}$, E. Pian ${ }^{3}$, A. Bulgarelli ${ }^{4}$, P. Caraveo ${ }^{5}$, R. F. Viotti ${ }^{1}$, M. F. Corcoran ${ }^{6}$, A. Giuliani ${ }^{5}$, C. Pittori $^{7}$, F. Verrecchia ${ }^{7}$, S. Vercellone ${ }^{5}$, S. Mereghetti ${ }^{5}$, A. Argan ${ }^{1}$, G. Barbiellini ${ }^{8}$, F. Boffelli ${ }^{9}$, P. W. Cattaneo ${ }^{9}$, A. W. Chen $^{5,10}$, V. Cocco ${ }^{1}$, F. D’Ammando ${ }^{1,2}$, E. Costa ${ }^{1}$, G. De Paris ${ }^{1}$, E. Del Monte ${ }^{1}$, G. Di Cocco ${ }^{4}$, I. Donnarumma ${ }^{1}$, Y. Evangelista ${ }^{1}$, A. Ferrari ${ }^{10,11}$, M. Feroci ${ }^{1}$, M. Fiorini ${ }^{5}$, T. Froysland ${ }^{2,10}$, F. Fuschino ${ }^{4}$, M. Galli ${ }^{12}$, F. Gianotti ${ }^{4}$, C. Labanti ${ }^{4}$, I. Lapshov ${ }^{1}$, F. Lazzarotto ${ }^{1}$, P. Lipari ${ }^{13}$, F. LonGo $^{8}$, M. Marisaldi ${ }^{4}$, M. Mastropietro ${ }^{14}$, E. Morelli ${ }^{4}$, E. Moretti ${ }^{8}$, A. Morselli ${ }^{15}$, L. Pacciani ${ }^{1}$, A. Pellizzoni ${ }^{16}$, F. Perotti ${ }^{5}$, G. Piano ${ }^{1,2,15}$, P. Picozza ${ }^{2,15}$, M. Pilia ${ }^{17}$, G. Porrovecchio ${ }^{1}$, G. Pucella ${ }^{1}$, M. Prest ${ }^{18}$, M. Rapisarda ${ }^{19}$, A. Rappoldi ${ }^{9}$, A. Rubini ${ }^{1}$, P. Soffitta ${ }^{1}$, M. Trifoglio ${ }^{4}$, A. Trois ${ }^{1}$, E. Vallazza ${ }^{8}$, V. Vittorini ${ }^{1,2}$, A. Zambra ${ }^{5}$, D. Zanello ${ }^{13}$, P. SAntolamazza ${ }^{7}$, P. Giommi ${ }^{7}$, S. Colafrancesco $^{7}$, L. A. ANTONELLi ${ }^{20}$, AND L. SALOTTI ${ }^{21}$

${ }^{1}$ INAF/IASF-Roma, I-00133 Roma, Italy

2 Dipartimento di Fisica, Univ. Tor Vergata, I-00133 Roma, Italy

${ }^{3}$ Osservatorio Astronomico di Trieste, Trieste, Italy

${ }^{4}$ INAF/IASF-Bologna, I-40129 Bologna, Italy

5 INAF/IASF-Milano, I-20133 Milano, Italy

${ }^{6}$ CRESST and Universities Space Research Association, NASA/Goddard Space Flight Center, Code 662, Greenbelt, MD 20771, USA

7 ASI Science Data Center, I-00044 Frascati (Roma), Italy

${ }^{8}$ Dipartimento di Fisica and INFN Trieste, I-34127 Trieste, Italy

${ }^{9}$ INFN-Pavia, I-27100 Pavia, Italy

${ }^{10}$ CIFS-Torino, I-10133 Torino, Italy

${ }^{11}$ Dipartimento di Fisica, Universitá di Torino, Turin, Italy

12 ENEA-Bologna, I-40129 Bologna, Italy

${ }^{13}$ INFN-Roma La Sapienza, I-00185 Roma, Italy

${ }^{14}$ CNR-IMIP, Roma, Italy

15 INFN Roma Tor Vergata, I-00133 Roma, Italy

16 INAF-Osservatorio Astronomico di Cagliari, localita' Poggio dei Pini, Strada 54, I-09012 Capoterra, Italy

${ }^{17}$ Dipartimento di Fisica, Universitá dell'Insubria, Via Valleggio 11, I-22100 Como, Italy

${ }^{18}$ Dipartimento di Fisica, Univ. Dell'Insubria, I-22100 Como, Italy

${ }^{19}$ ENEA Frascati, I-00044 Frascati (Roma), Italy

${ }^{20}$ INAF-Osservatorio Astron. di Roma, Monte Porzio Catone, Italy

21 Agenzia Spaziale Italiana, I-00198 Roma, Italy

Received 2009 March 2; accepted 2009 March 16; published 2009 June 4

\begin{abstract}
We present the results of extensive observations by the gamma-ray AGILE satellite of the Galactic region hosting the Carina nebula and the remarkable colliding wind binary Eta Carinae ( $\eta$ Car) during the period 2007 July-2009 January. We detect a gamma-ray source (1AGL J1043-5931) consistent with the position of $\eta$ Car. If 1AGL J1043-5931 is associated with the Car system, our data provide the long sought first detection above $100 \mathrm{MeV}$ of a colliding wind binary. The average gamma-ray flux above $100 \mathrm{MeV}$ and integrated over the preperiastron period 2007 July-2008 October is $F_{\gamma}=(37 \pm 5) \times 10^{-8} \mathrm{ph} \mathrm{cm}^{-2} \mathrm{~s}^{-1} \mathrm{cor}^{-}$ responding to an average gamma-ray luminosity of $L_{\gamma}=3.4 \times 10^{34} \mathrm{erg} \mathrm{s}^{-1}$ for a distance of $2.3 \mathrm{kpc}$. We also report a two-day gamma-ray flaring episode of 1AGL J1043-5931 on 2008 October 11-13 possibly related to a transient acceleration and radiation episode of the strongly variable shock in the system.
\end{abstract}

Key words: gamma rays: observations - stars: individual (Eta Carinae) - stars: winds, outflows - X-rays: binaries

\section{INTRODUCTION}

Eta Carinae ( $\eta$ Car) is a very massive $(\sim 100$ solar masses $)$ star known for its strong mass outflow eruptions, and is one of the most interesting objects of our Galaxy (e.g., Davidson \& Humphrey 1997). Radial velocity variations of spectroscopic lines accumulated over the years provide a strong evidence that $\eta$ Car is a binary system (e.g., Damineli et al. 2008a, 2008b) where the primary star is a luminous blue variable (LBV) star orbiting in a very eccentric binary $(e \sim 0.9)$ with a companion star believed to be an $\mathrm{O}$ star of $\sim 30$ solar masses. The orbital period is 5.53 years ( 2023 days; e.g., Damineli et al. 2008a): the system has been monitored in the radio, mm, IR, optical, and X-ray bands for at least three cycles. Both stars emit dense and high-velocity gaseous winds, and the binary system is ideal to study the interaction of colliding winds and to test theories of particle acceleration and radiation under extreme conditions. The mass outflow rates and wind speeds of the two stars inferred from the wealth of all available data are $\dot{M}_{1} \simeq 2 \times 10^{-4} M_{\odot} \mathrm{yr}^{-1}, \dot{M}_{2} \simeq 2 \times 10^{-5} M_{\odot} \mathrm{yr}^{-1}, v_{1} \simeq$ $600 \mathrm{~km} \mathrm{~s}^{-1}, v_{2} \simeq 3000 \mathrm{~km} \mathrm{~s}^{-1}$ (Pittard et al. 2005). $\eta$ Car is then interesting among other colliding wind binaries (CWBs) since the observable $\mathrm{X}$-ray emission in the $2-10 \mathrm{keV}$ band is almost entirely produced by the shocked fast wind of the secondary star, with little if any contribution from the slow shocked wind of $\eta$ Car itself. The system is known for its variability and occasional erratic eruptions detected in the IR and optical bands, as well as for its distinct asymmetric pattern of optical line and X-ray emission during its orbital period (Corcoran et al. 2001).

$\eta$ Car has been repeatedly observed in the energy ranges $1-10 \mathrm{keV}$ and $20-100 \mathrm{keV}$ by different observatories. It is 
Table 1

AGILE Observations of the $\eta$ Car Region

\begin{tabular}{|c|c|c|c|c|c|c|c|}
\hline$A G I L E$ Orbits & Date Interval & MJD & No. of Days & $\begin{array}{c}\eta \text { Car } \\
\text { phase }^{\text {a }}\end{array}$ & $\sqrt{T S} \mathrm{~b}$ & Counts & Average flux ${ }^{\mathrm{c}}$ \\
\hline $1146-1299$ & 2007.07.13-2007.07.24 & $54294.5-54305.5$ & 11 & 0.732 & 4.1 & $105 \pm 29$ & $67 \pm 18$ \\
\hline 1584-1708 & 2007.08.13-2007.08.22 & $54325.5-54334.5$ & 9 & 0.747 & 1.1 & $<73$ & $<67$ \\
\hline $3673-4009$ & 2008.01.08-2008.02.01 & $54473.5-54497.5$ & 24 & 0.824 & 4.1 & $166 \pm 45$ & $55 \pm 14$ \\
\hline $6778-7001$ & 2008.08.15-2008.08.31 & $54693.5-54709.5$ & 16 & 0.930 & 1.3 & $<105$ & $<46$ \\
\hline $7569-7664$ & 2008.10.10-2008.10.17 & $54749.5-54756.5$ & 7 & 0.956 & 4.0 & $80 \pm 23$ & $99 \pm 28^{d}$ \\
\hline 8899-8995 & 2009.01.12-2009.01.19 & $54843.5-54850.5$ & 7 & 0.002 & 2.2 & $48 \pm 22$ & $<94$ \\
\hline
\end{tabular}

Notes.

a Average orbital phase of $\eta$ Car calculated at the center of the time interval.

b Square root of the Maximum Likelihood Test Statistic (TS) representing the statistical significance of the detection.

c Gamma-ray flux of 1AGL J1043-5931 above $100 \mathrm{MeV}$ in units of $10^{-8} \mathrm{ph} \mathrm{cm}^{-2} \mathrm{~s}^{-1}$ obtained by taking into account the nearby source AGL J1046-5832 in the multisource likelihood analysis. We also indicate $2 \sigma$ upper limits in the same units.

${ }^{\mathrm{d}}$ During this period the source reached the gamma-ray flux above $100 \mathrm{MeV}$ of $F=(270 \pm 65) \times 10^{-8} \mathrm{ph}^{-2} \mathrm{~s}^{-1}$.

certainly the only source showing a nonthermal X-ray spectrum within a region centered on $\eta$ Car with a $1^{\circ}$ diameter (the anomalous X-ray pulsar AXP 1E $1048.1-5937$ is about 0.6 away). Whereas the $1-10 \mathrm{keV}$ spectrum is dominated by a quasithermal and variable component (Corcoran et al. 2001; Corcoran 2005; Viotti et al. 2002), the hard X-ray observations show nonthermal emission that appears to vary along the orbit (Viotti et al. 2004; Leyder et al. 2008). The $\eta$ Car source was detected with high significance by BSAX-PDS and INTEGRAL-ISGRI far from periastron. INTEGRAL is capable of resolving field sources with a few arcminute resolution in the hard X-ray range. Although INTEGRAL observed the system at different phase periods (0.99-0.01, 0.16-0.19, 0.35-0.37), $\eta$ Car was detected only during the phase interval $0.16-0.19$ with an average 22-100 keV X-ray flux of $F=1.1 \times 10^{-11} \mathrm{erg} \mathrm{cm}^{-2} \mathrm{~s}^{-1}$ (Leyder et al. 2008).

The Carina region has been observed at gamma-ray energies above a few MeV by the OSSE, COMPTEL, and EGRET instruments on board the Compton Gamma-Ray Observatory (CGRO). An EGRET gamma-ray source (3EG J1048-5840) is cataloged at about $1^{\circ}$ distance from $\eta$ Car. However, no gammaray emission above $100 \mathrm{MeV}$ has been reported by CGRO from the $\eta$ Car region. ${ }^{22}$

The gamma-ray astrophysics mission AGILE (Tavani et al. 2008) observed several times the Carina region in the Galactic plane during its early operational phases and Cycle-1 observations. Here we report the main results of the gamma-ray observations of the $\eta$ Car region carried out by the AGILE satellite during the period 2007 July-2009 January, simultaneously in the energy bands $30 \mathrm{MeV}-30 \mathrm{GeV}$ and $18-60 \mathrm{keV}$. A high-confidence gamma-ray source (1AGL J1043-5931) was detected positionally consistent with $\eta$ Car by integrating all data, as well as by considering specific observation periods.

\section{AGILE 2007-2008 OBSERVATIONS}

The AGILE mission has been operating since 2007 April (Tavani et al. 2008). The AGILE scientific instrument is very compact and is characterized by two co-aligned imaging

22 EGRET observed the $\eta$ Car field several times (see, e.g., Table 2 of Kaspi et al. 2000). Observations close to periastron were VP32 (1992 June 25-July 02, phase 0.014) and VP630 (1997 September 22-October 6, phase 0.963; Hartman et al. 1999; Kaspi et al. 2000). detectors operating in the energy ranges $30 \mathrm{MeV}-30 \mathrm{GeV}$ (Gamma-Ray Imaging Detector (GRID); Barbiellini et al. 2002; Prest et al. 2003) and 18-60 keV (Super-AGILE; Feroci et al. 2007), as well as by an anticoincidence system (Perotti et al. 2006) and a calorimeter (Labanti et al. 2006). AGILE's performance is characterized by large fields of view (FOV; 2.5 and $1 \mathrm{sr}$ for the gamma-ray and hard X-ray bands, respectively), optimal angular resolution, and good sensitivity (see Tavani et al. 2008 for details about the mission and main instrument performance). Flux sensitivity for a typical 1 week observing period can reach the level of several tens of $10^{-8} \mathrm{ph} \mathrm{cm}^{-2} \mathrm{~s}^{-1}$ above $100 \mathrm{MeV}$, and $10-20 \mathrm{mCrab}$ in the $18-60 \mathrm{keV}$ range depending on off-axis angles and pointing directions.

The AGILE satellite repeatedly pointed at the Carina region for a total of $\sim 130$ days during the time period 2007 July-2009 January. Table 1 summarizes the AGILE observations of the field. Analysis of the gamma-ray data was carried out with the $F T 3 a b_{2}$ calibrated filter, with a gamma-ray event selection that takes into account standard South Atlantic Anomaly event cuts and $80^{\circ}$ Earth albedo filtering. We used the AGILE gamma-ray software release Build 17 and the standard hard X-ray analysis software.

Figure 1 shows the integrated sky map of the $\eta$ Car region at energies above $100 \mathrm{MeV}$ for the period 2007 July-2008 October. A gamma-ray source is detected with high confidence $(7.8 \sigma)$ at the position $(l, b)=287.6,-0.7 \pm 0.3$ (stat) \pm 0.1 (syst). The average gamma-ray flux above $100 \mathrm{MeV}$ and integrated over the whole period 2007 July-2008 October is $F_{\gamma}=$ $(37 \pm 5) \times 10^{-8} \mathrm{ph} \mathrm{cm}^{-2} \mathrm{~s}^{-1}$. We call this source ${ }^{23}$ 1AGL J1043-5931 following the source designation of the first AGILE catalog of high-confidence gamma-ray sources (Pittori et al. 2009). We included in all our multisource analysis the nearby gamma-ray source ${ }^{24}$ AGL J1046-5832 that the AGILE-GRID detects with an average and constant gamma-ray flux above $100 \mathrm{MeV}$ of $f_{\gamma}=(27 \pm 4) \times 10^{-8} \mathrm{ph} \mathrm{cm}^{-2} \mathrm{~s}^{-1} . \eta$ Car is well within the $95 \%$ confidence radius gamma-ray error box of 1AGL J1043-5931; the other nearby hard X-ray sources in

\footnotetext{
23 The same source is also listed in the Fermi Bright Source Catalogs as OFGL J1045.6-5937 (Abdo et al. 2009).

24 This source, which did not reach the stringent significance threshold of the First AGILE Catalog (Pittori et al. 2009), is included in the Fermi Bright Source List as 0FGL J1047.6-5834 and is identified with PSR J1048-5832 (Abdo et al. 2009).
} 


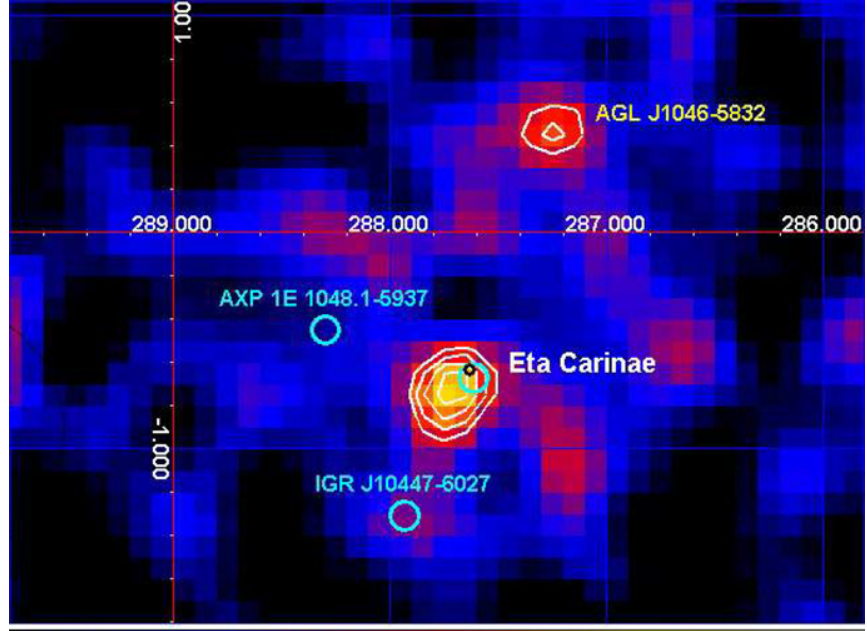

$\begin{array}{llllllll}0.00035 & 0.0004 & 0.00045 & 0.0005 & 0.00055 & 0.0006 & 0.00065 & 0.0007\end{array}$

Figure 1. AGILE gamma-ray intensity map in Galactic coordinates of the $\eta$ Car region above $100 \mathrm{MeV}$ summing all data collected from 2007 July to 2008 October. The central gamma-ray source that can be associated with $\eta$ Car is 1AGL J1043-5931; we also indicate the prominent nearby gamma-ray source AGL J1046-5832 which is associated with the radio pulsar PSR B104658 (Kaspi et al. 2000; Abdo et al. 2009). The color bar scale is in units of photons $\mathrm{cm}^{-2} \mathrm{~s}^{-1}$ pixel $^{-1}$. Pixel size is 0.1 , and we used a three-bin Gaussian smoothing. White contour levels of the AGILE sources start from 0.0005 and increase in steps of 0.000028 . The optical position of $\eta$ Car is marked by a small black circle. The INTEGRAL sources (Leyder et al. 2008) are marked with cyan circles.

the field (the anomalous X-ray pulsar AXP 1E 1048.1-5937, and IGR J10447-6027) are excluded. The Super-AGILE hard
X-ray imager did not detect a source coincident with 1AGL J1043-5931 for both short and long integrations of Table 1 . Depending on the source position in the FOV, the typical $3 \sigma$ Super-AGILE upper limit is $10-20 \mathrm{mCrab}$, i.e., consistent with the INTEGRAL detection and upper limits of $\eta$ Car.

We searched for short timescale variability of the gammaray and hard X-ray flux from 1AGL J1043-5931 throughout the whole AGILE observing periods of the Carina region. A 2-day gamma-ray flare from the direction of $\eta$ Car was detected during the observation of 2008 October 10-17. The emission reached its peak gamma-ray emission during the period 2008 October 11 (02:57 UT)-2008 October 13 (04:16 UT). Our analysis gives a $5.2 \sigma$ detection of a source at the position $(l, b)=288.0,-0.4 \pm 0.6$ fully consistent with the 1AGL J1043-5931 position but with a gamma-ray flux above $100 \mathrm{MeV}$ of $F=(270 \pm 65) \times 10^{-8} \mathrm{ph} \mathrm{cm}^{-2} \mathrm{~s}^{-1}$. Figure 2 shows the time sequence of 2-day integration gamma-ray maps of the region during the period 2008 October 10-17.

Figure 3 shows the AGILE gamma-ray data of Table 1 superimposed with the simultaneous Rossi X-Ray Timing Explorer (RXTE; PCU2 net rate) light curve in the energy band 2-15 keV during the period 2007 February-2009 January (the typical and relatively abrupt decrease of the X-ray emission near periastron is clearly visible).

Figure 4 shows two representative different broadband spectral states of 1AGL J1043-5931 obtained during the period 2007 July-2008 October together with the historical X-ray and hard X-ray data reported from $\eta$ Car. We mark in blue the average spectrum obtained by integrating all data outside periastron, and with a red cross the flux corresponding to the flaring state of 2008 October 11-13. We also report in the same plot the
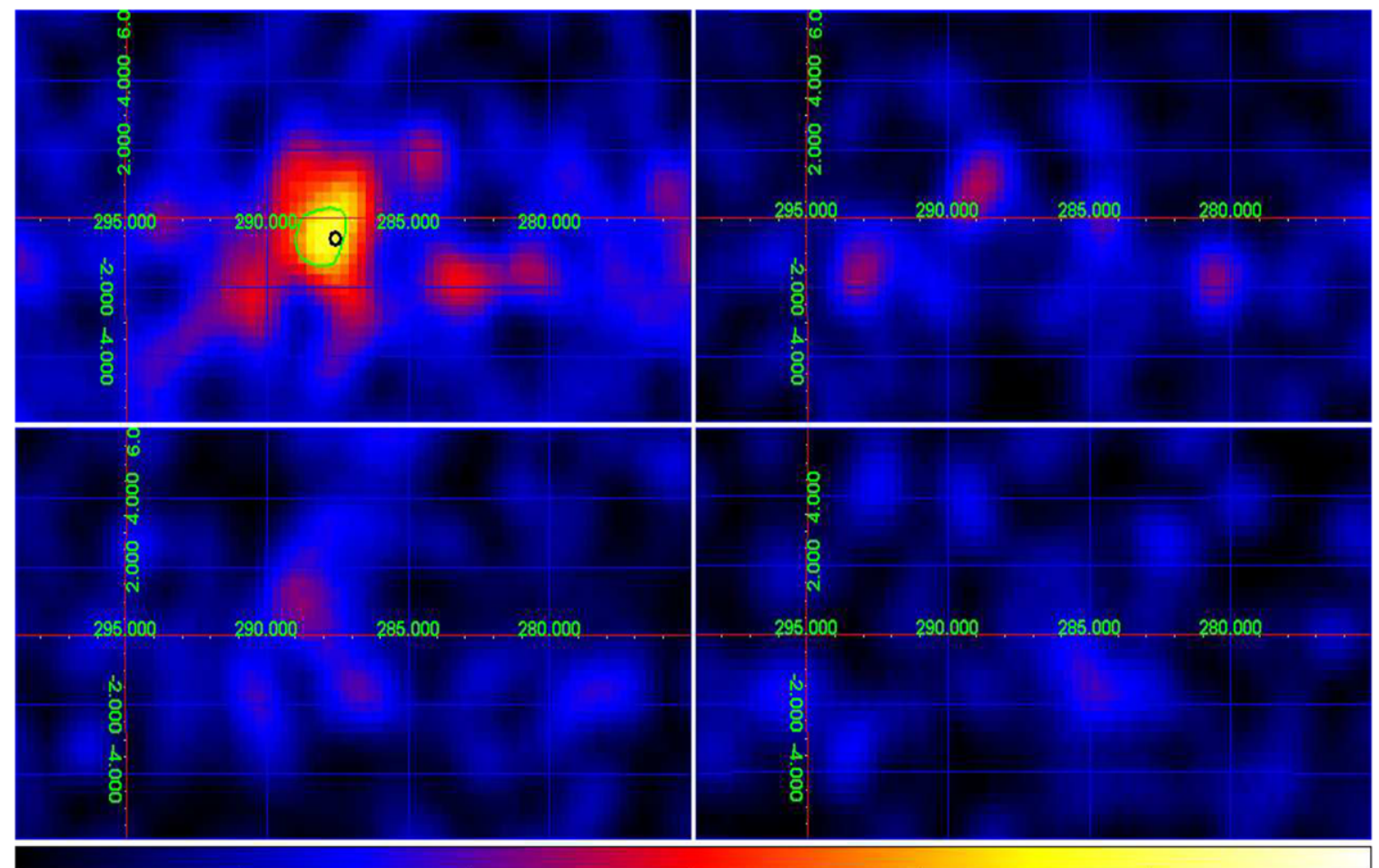

0.1

0.2

0.3

0.5

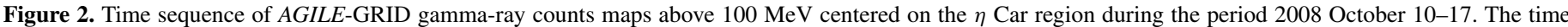

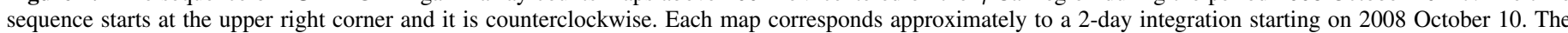

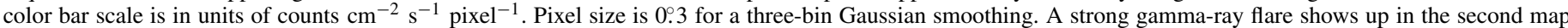

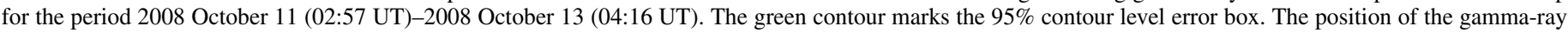
flaring source is consistent with the 1AGL J1043-5931 position and with $\eta$ Car (marked by a black small circle). 


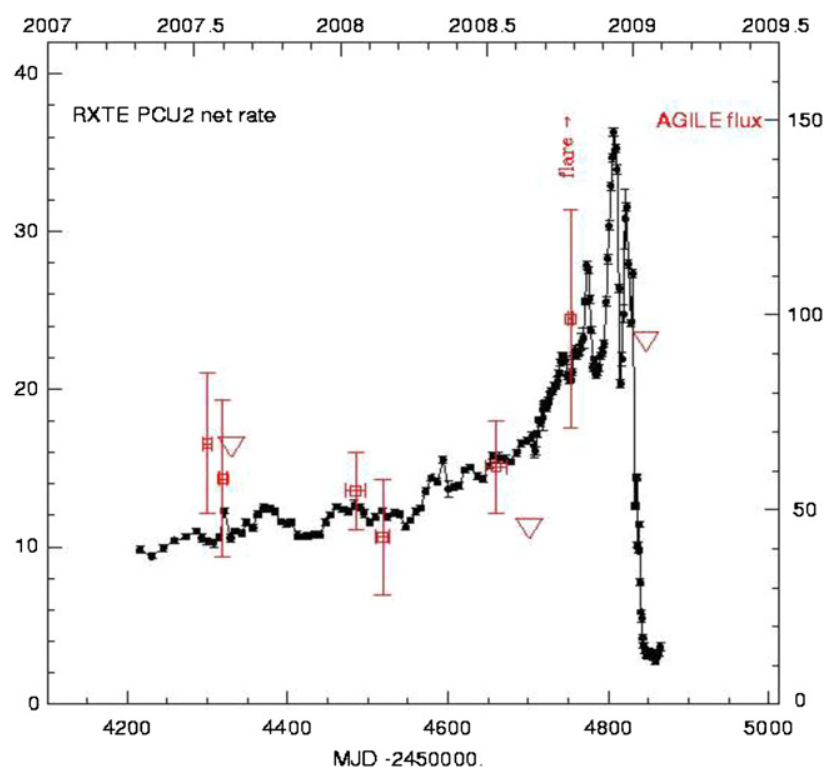

Figure 3. AGILE gamma-ray light curve of 1AGL J1043-5931 showing the fluxes above $100 \mathrm{MeV}$ (right axis scale in units of $10^{-8} \mathrm{ph} \mathrm{cm}^{-2} \mathrm{~s}^{-1}$ ) averaged over the observing periods of Table 1 (red crosses) and superimposed with the RXTE PCU2 net rate X-ray light curve of $\eta$ Car (black symbols, left axis) obtained during the dedicated campaign observing the last cycle and periastron passage. Triangles indicate $2 \sigma$ upper limits. We also mark the occurrence of the 2008 October 11-13 flare when the source reached a gamma ray flux above $100 \mathrm{MeV}$ of $F=(270 \pm 65) \times 10^{-8} \mathrm{ph} \mathrm{cm}^{-2} \mathrm{~s}^{-1}$.

(nonsimultaneous) BSAX-MECS and the INTEGRAL-ISGRI spectral states of $\eta$ Car reported in the literature (Viotti et al. 2004; Leyder et al. 2008). It is interesting to note that if $1 \mathrm{AGL}$ $\mathrm{J} 1043-5931$ is associated with $\eta$ Car, the average AGILE spectrum together with the INTEGRAL historical spectrum is in qualitative agreement with expectations based on inverse Compton (IC) and/or pion decay models of gamma-ray emission from CWBs (e.g., Reimer et al. 2006). For the 2008 October 11-13 flaring episode, the Super-AGILE 18-60 keV upper limit is $70 \mathrm{mCrab}$ in the energy band $18-60 \mathrm{keV}$. Obtaining simultaneous hard X-ray and gamma-ray data during the flaring state of 1AGL J1043-5931 is crucial to study the broadband variability of the source. However, due to unfavorable source positioning of 1AGL J1043-5931 in the Super-A FOV in mid-October 2008, the hard X-ray upper limit is not very constraining.

AGILE pointed at the Carina Region during the period 2009 January 12-19 as a special repointing to cover the $\eta$ Car periastron passage (calculated to be occurring on 2009 January 11). We cannot exclude, at this stage, the existence of a weak gamma-ray source consistent with 1AGL J1043-5931. However, we can currently provide a $2 \sigma$ upper limit to the emission above $100 \mathrm{MeV}$ of $94 \times 10^{-8} \mathrm{ph} \mathrm{cm}^{-2} \mathrm{~s}^{-1}$. A more detailed analysis of the AGILE and multifrequency data during the $\eta$ Car periastron passage will be discussed elsewhere.

\section{DISCUSSION}

$\eta$ Car is located in the Carina nebula that extends for several degrees in a Galactic region characterized by dense molecular clouds, young stars, and star formation sites. However, within the 1AGL J1043-5931 error box, $\eta$ Car itself is by far the strongest and hardest source in the $2-10 \mathrm{keV}$ and $22-$ $100 \mathrm{keV}$ ranges. Another source inside the AGILE error box and 7 arcmin away from $\eta$ Car is the X-ray binary HD 93162/ WR 25 (WN6+O4, with an orbital period of 208 days; Gamen

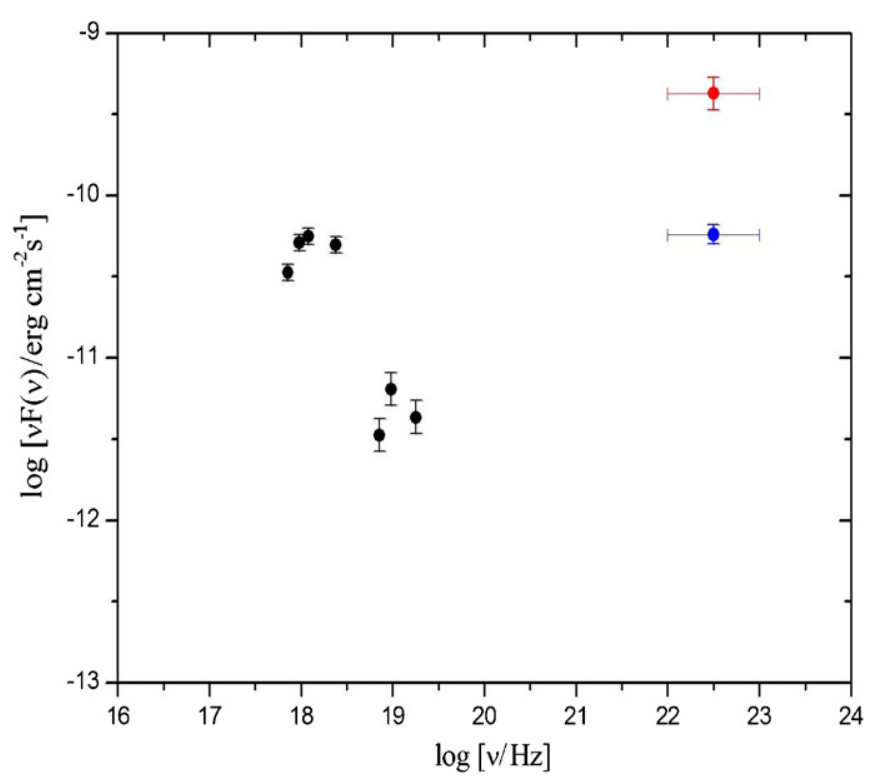

Figure 4. Combined spectral power flux of $\eta$ Car as reported by the SAXMECS in the energy range 1-10 keV (phase 0.46; Viotti et al. 2004), by INTEGRAL in the energy range $22-100 \mathrm{keV}$ (phase $0.16-0.19$; Leyder et al. 2008) plotted together with the two broadband gamma-ray spectral states of 1AGL J1043-5931 measured by AGILE during the period 2007 July-2008 October ( $\eta$ Car phase 0.73-0.95). The lower blue point marks the average gamma-ray spectral flux, and the upper red point indicates the spectral state during the gamma-ray flare of 2008 October 11-13.

et al. 2006). This system is known to be a colliding wind system. Pollock \& Corcoran (2006) found significant variability, possibly periodic, of its X-ray flux. However, WR 25 was not detected in the hard X-ray range by INTEGRAL during any of its observations. Furthermore, no other prominent hard X-ray source is known in the 1AGL J1043-5931 error box except for $\eta$ Car itself. The two nearby hard X-ray sources detected by INTEGRAL (Leyder et al. 2008) are outside the $95 \%$ confidence level error box of AGILE. Multiple gamma-ray sources within the 1AGL J1043-5931 error box cannot be excluded but are unlikely.

Based on the accumulated multifrequency evidence and the nature of the source, we consider the association of 1AGL J1043-5931 and $\eta$ Car as very likely. We briefly elaborate below on the theoretical implications of our results assuming that 1AGL J1043-5931 is indeed the gamma-ray counterpart of $\eta$ Car.

CWBs are ideal systems to test theories of hydrodynamical shocks and particle acceleration under extreme radiative conditions provided by the proximity of the two stars. In particular, supersonic winds can form efficient shocks where electrons and protons can be accelerated through first-order Fermi (Eichler \& Usov 1993) or other acceleration mechanisms. IC scattering of shock-accelerated particles in the presence of the very intense IR-optical-UV background of the nearby very bright stars provides a crucial ingredient in CWBs. In addition to synchrotron and bremsstrahlung electron emissions, the IC emission can dominate the high energy spectrum at energies larger than several tens of $\mathrm{keV}$ up to $\mathrm{MeV}-\mathrm{GeV}$ energies. Furthermore, if protons are efficiently accelerated, they can interact with the dense stellar outflows and produce gamma-rays by pion production and neutral pion decay (e.g., Eichler \& Usov 1993; Benaglia \& Romero 2003; Reimer et al. 2006). All these ingredients are important for the $\eta$ Car system and detailed hydrodynamical modeling of the mass outflow have been developed (Parkin \& 
Pittard 2008; Parkin et al. 2009; Okazaki et al. 2008). A comprehensive and detailed theoretical analysis of our data is beyond the scope of this Letter. We outline here a few important points.

If 1AGL J1043-5931 is the $\eta$ Car gamma-ray counterpart, our data show the first remarkable detection of a colliding wind system at hundreds of $\mathrm{MeV}$ energies, confirming the efficient particle acceleration and the highly nonthermal nature of the strong shock in a CWB. The average gamma-ray flux of 1AGL J1043-5931 translates into gamma-ray luminosity of $L_{\gamma}=3.4 \times 10^{34} \mathrm{erg} \mathrm{s}^{-1}$ for an $\eta$ Car distance of $2.3 \mathrm{kpc}$ (Smith 2006), corresponding to a fraction of a percent of the total wind kinetic power. The 2008 October 11-13 flare episode has a luminosity of $L_{\gamma}=2 . \times 10^{35} \mathrm{erg} \mathrm{s}^{-1}$. The average broadband gamma-ray spectrum determined by AGILE is in qualitative agreement with expectations of CWB spectra as calculated for dominant IC and neutral pion decay processes (Benaglia \& Romero 2003; Reimer et al. 2006).

While the gamma-ray flux of 1AGL J1043-5931 is roughly constant during the time span covered by our observations, a significant variability was detected on a few day timescale in 2008 October. This episode indicates that the gamma-ray emission can be associated with intermittent strong shock acceleration episodes and/or magnetic field enhancements to be expected for a very variable and inhomogeneous mass outflow from the stars of the $\eta$ Car system. In particular, we note that the strong gamma-ray flaring episode occurred a few months before periastron, when the efficiency of transforming a mass outflow enhancement into particle acceleration is expected to increase because of the closeness of the two stars. $\eta$ Car provides then some crucial ingredients regarding the formation of high-energy emission in CWBs: (1) strong variability of the mass outflows; (2) a high-speed wind from the less massive companion; (3) a radiative environment with a specific bath of soft photons from both stars (IR, optical, and UV fluxes) that can illuminate the shock region and provide a time variable environment for enhanced IC emission in the $100 \mathrm{MeV}$ range and beyond. The theoretical implications are far reaching.
The $\eta$ Car system would provide the first CWB to test the particle acceleration models for nonrelativistic mass outflows under a specific set of physical conditions. It is very important to assess the efficiency of the particle acceleration process in such a radiative environment. A gamma-ray flaring episode lasting $\sim 2$ days implies a fast acceleration timescale and subsequent radiation and decay of the strong shock properties leading to the efficient emission. If the gamma-ray emission is associated with $\eta$ Car, our observations provide important data to test shock acceleration models. Future gamma-ray observations and analysis will further contribute to enlighten the emission mechanism and the ultimate origin of 1AGL J1043-5931.

\section{REFERENCES}

Abdo, A. A., et al. 2009, http://fermi.gsfc.nasa.gov

Barbiellini, G., et al. 2002, Nucl. Instrum. Methods Phys. Res. A, 490, 146

Benaglia, P., \& Romero, G. E. 2003, A\&A, 399, 1121

Corcoran, M. F. 2005, AJ, 129, 2018

Corcoran, M. F., et al. 2001, ApJ, 547, 1034

Damineli, A., et al. 2008a, MNRAS, 384, 1649

Damineli, A., et al. 2008b, MNRAS, 386, 2330

Davidson, K., \& Humphrey, R. M. 1997, ARA\&A, 53, 1

Eichler, D., \& Usov, V. 1993, ApJ, 402, 271

Feroci, M., et al. 2007, Nucl. Instrum. Methods Phys. Res. A, 581, 728

Gamen, R., et al. 2006, A\&A, 460, 777

Hartman, R. C., et al. 1999, ApJS, 123, 79

Kaspi, V. M., et al. 2000, ApJ, 528, 445

Labanti, C., et al. 2006, Proc. SPIE, 6266, 62663

Leyder, J.-C., Walter, R., \& Rauw, G. 2008, A\&A, 477, L29

Okazaki, A. T., et al. 2008, MNRAS, 388, L39

Parkin, E. R., \& Pittard, J. M. 2008, MNRAS, 388, 1047

Parkin, E. R., et al. 2009, MNRAS, in press (arXiv:0901.0862v1)

Perotti, F., et al. 2006, Nucl. Instrum. Methods Phys. Res. A, 556, 228

Pittard, J. M., et al. 2005, http://www.aoc.nrao.edu/events/xraydio

Pittori, C., et al. 2009, A\&A, submitted (http://agile.asdc.asi.it)

Pollock, A. M., \& Corcoran, M. F. 2006, A\&A, 445, 1093

Prest, M., et al. 2003, Nucl. Instrum. Methods Phys. Res. A, 501, 280

Reimer, A., Pohl, M., \& Reimer, O. 2006, ApJ, 644, 1118

Smith, N. 2006, ApJ, 644, 1151

Tavani, M., et al. 2008, A\&A, in press (arXiv:0807.4254)

Viotti, R. F., et al. 2002, A\&A, 385, 874

Viotti, R. F., et al. 2004, A\&A, 420, 527 